\title{
COMMENTARY
}

\section{Activated partial thromboplastin time waveform analysis as specific sepsis marker in cardiopulmonary bypass surgery}

\author{
Christian P Schneider*, Martin K Angele and Wolfgang H Hartl \\ See related research by Delannoy et al., http://ccforum.com/content/13/6/R180
}

\begin{abstract}
Throughout the last years, several new diagnostic biomarkers have been introduced into clinical routine to identify a systemic inflammatory response syndrome (SIRS) or a septic state and to discriminate between these two entities. According to studies in selected patients, measurement of these biomarkers may be advantageous under certain clinical conditions. On an individual basis, however, these sepsis markers usually lack an adequate negative or positive predictive power. Therefore, physicians in charge still have to rely on a combination of personal experience and results from clinical or laboratory tests when deciding on a patient's therapy. For surgical patients, a key problem consists of the time delay which is associated with the diagnosis of serious postoperative infections and which may negatively affect outcome. It is in this context where the activated partial thromboplastin time waveform analysis may represent a promising new method to discriminate between SIRS and sepsis, thereby shortening the time to therapy. Nevertheless, studies involving large patient populations will be necessary to prove the efficacy of this new diagnostic concept either as a single tool or in combination with the measurement of other biomarkers.
\end{abstract}

Although our understanding of the pathophysiology of sepsis and of subsequently derived therapeutic approaches has advanced significantly in the past, mortality of sepsis still remains unacceptably high. To improve this dilemma, diagnostic laboratory tests are urgently required that

*Correspondence: christian.schneider@med.uni-muenchen.de Department of Surgery, Munich University Hospital, Campus Großhadern, Ludwig-Maximilians-University, Marchioninistraße 15, 80933 Munich, Germany possess a high degree of accuracy and for which the results can be obtained in a very short time. For patients after cardiopulmonary bypass surgery, a recent publication demonstrated that an activated partial thromboplastin time biphasic waveform (BPW) analysis was able to discriminate between sepsis and nonseptic systemic inflammatory response syndrome (SIRS) with a sensitivity of $100 \%$ and a specificity of $93 \%$ [1]. In all patients, pneumonia was the septic focus. Compared with BPW analysis, the specificity and sensitivity of C-reactive protein (CRP) and procalcitonin (PCT) were inferior with respect to pneumonia prediction.

Delannoy and colleagues showed that perioperative complications such as haemorrhage, tamponade or reoperation were all associated with the emergence of postoperative SIRS and sepsis [1]. For cardiac surgical patients, these complications are also known to be important predictors for prolonged invasive ventilation [2]. Those observations are in line with the commonly accepted hypothesis that a large tissue trauma or prolonged states of shock initially lead to an increased inflammatory response that is followed by a state of immunosuppression, thereby increasing the susceptibility to infectious complications [3]. In these patients, however, the early and timely differentiation between SIRS and sepsis remains difficult. If an infectious focus becomes clinically evident, it will usually be too late to prevent the beginning of the septic vicious circle (for example, by initiating specific therapies such as antibiotics or surgical interventions) [4].

Postoperative fever is a key symptom requesting an urgent search for the underlying cause. Physicians caring for patients in intensive care units (ICUs) usually have a thorough knowledge of common infectious and noninfectious mechanisms that may cause fever in their patients. Many patients who are actually suffering from a septic focus, however, do not become febrile at the same time (for example, elderly patients or patients with uraemia). In those septic patients, the absence of secondary fever or 
of a general acute-phase response cannot be regarded as a favourable prognostic sign, but rather reflects the immunosuppressive phase of sepsis being associated with a high mortality. Other early manifestations of sepsis include a minor increase or decrease in the white-cell count or neutrophil percentage, a subtle change of mental status, or an elevated blood glucose level.

Early recognition of a septic state and of the septic focus is essential for successful treatment. The time to diagnosis, however, is often prolonged because key indicators (fever) are either missing, or have low specificity and sensitivity (leukocyte count), or can be only registered with a significant time delay (results from microbiological cultures). Because of these weaknesses, the availability of new biomarkers (PCT and IL-6) raised high hopes for all those caring for septic patients. Throughout the past years, the accuracy, predictive power and clinical utility of those biomarkers has been studied extensively.

A meta-analysis of 33 studies concluded that PCT constitutes an excellent laboratory marker for the discrimination between SIRS and sepsis [5]. When assessing the risk of patients with SIRS to develop sepsis, these authors found a global odds ratio of 15.6 when PCT concentrations were elevated, whereas the odds ratio was only 5.4 with increased CRP levels. The optimal decisional cut-off point varied between 0.6 and $5 \mathrm{ng} / \mathrm{l}$ when PCT was analysed, whereas corresponding variations were clearly wider for CRP (39 to $180 \mathrm{mg} / \mathrm{l}$ ). A similar superiority with respect to postoperative risk prediction was also found exclusively in surgical patients [6], where the accuracy of PCT even surmounted that of IL-6.

In selected patient populations, however, including immunosuppressed or very old patients, the diagnostic performance of PCT seems to vary $[7,8]$. In our own patient population, we made similar observations. Some patients had a normal PCT concentration after solid organ transplantation, although there was unequivocal evidence for a serious infection. This disease-specific PCT reaction may be one of the reasons for the inconclusive results from another systematic review published recently [9]. Nevertheless, most authors found PCT to be superior to CRP or IL-6 $[10,11]$. It should be noted that the use of CRP as a diagnostic tool is not completely unhelpful. Studies focusing selectively on CRP attested acceptable reliability, with a sensitivity of $85 \%$ and a specificity of about $70 \%$. CRP measurement therefore remains an option when PCT testing is not available [12].

Downey and colleagues were the first to discover an abnormality of the optical transmission waveform obtained during measurement of the activated partial thromboplastin time (reviewed in [13]). This abnormality, a BPW, is caused by in vitro formation of calcium-induced complexes between very-low-density lipoproteins and CRP. Waveform analysis is a rapid and inexpensive method, being part of the routine activated partial thromboplastin time measurement. BPW analysis was originally introduced as a marker for disseminated intravascular coagulation. Subsequent studies identified BPW analysis as an indicator of a systemic inflammatory response, as observed in sepsis [13]. The present study by Delannoy and colleagues demonstrated that BPW analysis was able to discriminate between sepsis and nonseptic SIRS patients with a sensitivity of $100 \%$ and a specificity of $93 \%$ (threshold value of $0.456 \% \mathrm{~T} / \mathrm{second}$ ). Only 32 cardiac surgery patients, however, were included [1].

An earlier investigation by Chopin and colleagues studied 187 patients with 217 episodes of SIRS, of which 34 patients were classified with sepsis, 26 patients with severe sepsis, and 50 patients with septic shock. The diagnostic sensitivity and specificity of BPW analysis for the combined group of severe sepsis and septic shock were $92 \%$ and $67 \%$, respectively [14]. In another recent study in ICU patients, the sensitivity of BPW analysis for sepsis was $81 \%$, with a specificity of $76 \%$. The combination of BPW analysis with PCT measurement did not increase the sensitivity but raised the specificity to $94 \%$ [15]. The use of BPW analysis, however, is not limited to ICU patients. Smith and colleagues found that non-ICU patients are more likely to have positive blood cultures when waveform analysis yields abnormal results [16].

BPW analysis therefore appears to be a promising parameter for the discrimination between SIRS and sepsis. To corroborate the use of BPW analysis, however, further studies in heterogeneous patient populations including larger patient numbers are required. Only if the precise diagnostic significance is established in relation to the underlying disease will BPW analysis become an important component in the routine panel of sepsis markers currently used.

\section{Abbreviations}

$\mathrm{BPW}=$ biphasic waveform; $\mathrm{CRP}=\mathrm{C}$-reactive protein; $\mathrm{ICU}=$ intensive care unit; $\mathrm{IL}=$ interleukin; $\mathrm{PCT}=$ procalcitonin; SIRS = systemic inflammatory response syndrome.

\section{Competing interests}

The authors declare that they have no competing interests.

Published: 21 January 2010

\section{References}

1. Delannoy B, Guye ML, Slaiman DH, Lehot JJ, Cannesson M: Effect of cardiopulmonary bypass on activated partial thromboplastin time waveform analysis, serum procalcitonin and C-reactive protein concentrations. Crit Care 2009, 13:R180.

2. Cislaghi F, Condemi AM, Corona A: Predictors of prolonged mechanical ventilation in a cohort of 5123 cardiac surgical patients. Eur J Anaesthesiol 2009, 26:396-403.

3. Lenz A, Franklin GA, Cheadle WG: Systemic inflammation after trauma. Injury 2007, 38:1336-1345

4. Dellinger RP, Levy MM, Carlet JM, Bion J, Parker MM, Jaeschke R, Reinhart K, Angus DC, Brun-Buisson C, Beale R, Calandra T, Dhainaut JF, Gerlach H, Harvey M, Marini JJ, Marshall J, Ranieri M, Ramsay G, Sevransky J, Thompson BT, Townsend S, Vender JS, Zimmerman JL, Vincent JL; International Surviving 
Sepsis Campaign Guidelines Committee; American Association of CriticalCare Nurses; American College of Chest Physicians; American College of Emergency Physicians; Canadian Critical Care Society; European Society of Clinical Microbiology and Infectious Diseases; European Society of Intensive Care Medicine; European Respiratory Society; International Sepsis Forum; Japanese Association for Acute Medicine; Japanese Society of Intensive Care Medicine; Society of Critical Care Medicine; Society of Hospital Medicine; Surgical Infection Society; World Federation of Societies of Intensive and Critical Care Medicine: Surviving Sepsis Campaign: international guidelines for management of severe sepsis and septic shock: 2008. Crit Care Med 2008, 36:296-327.

5. Uzzan B, Cohen R, Nicolas P, Cucherat M, Perret GY: Procalcitonin as a diagnostic test for sepsis in critically ill adults and after surgery or trauma: a systematic review and meta-analysis. Crit Care Med 2006 34:1996-2003.

6. Schneider CP, Yilmaz Y, Kleespies A, Jauch KW, Hartl WH: Accuracy of procalcitonin for outcome prediction in unselected postoperative critically ill patients. Shock 2009, 31:568-673.

7. Dornbusch HJ, Strenger V, Kerbl R, Lackner H, Schwinger W, Sovinz P, Urban C: Procalcitonin and $\mathrm{C}$-reactive protein do not discriminate between febrile reaction to anti-T-lymphocyte antibodies and Gram-negative sepsis. Bone Marrow Transplant 2003, 32:941-945

8. Stucker F, Herrmann F, Graf JD, Michel JP, Krause KH, Gavazzi G: Procalcitonin and infection in elderly patients. J Am Geriatr Soc 2005, 53:1392-1395.

9. Tang BM, Eslick GD, Craig JC, McLean AS: Accuracy of procalcitonin for sepsis diagnosis in critically ill patients: systematic review and metaanalysis. Lancet Infect Dis 2007, 7:210-217.

10. Brunkhorst FM, Eberhard OK, Brunkhorst R: Discrimination of infectious and noninfectious causes of early acute respiratory distress syndrome by procalcitonin. Crit Care Med 1999, 27:2172-2176.
11. Aikawa N, Fujishima S, Endo S, Sekine I, Kogawa K, Yamamoto Y, Kushimoto S, Yukioka H, Kato N, Totsuka K, Kikuchi K, Ikeda T, Ikeda K, Harada K, Satomura S: Multicenter prospective study of procalcitonin as an indicator of sepsis. $J$ Infect Chemother 2005, 11:152-159.

12. Sierra R, Rello J, Bailén MA, Benítez E, Gordillo A, León C, Pedraza S: C-reactive protein used as an early indicator of infection in patients with systemic inflammatory response syndrome. Intensive Care Med 2004, 30:2038-2045.

13. Dempfle CE, Borggrefe M: The hidden sepsis marker: aPTT waveform analysis. Thromb Haemost 2008, 100:9-10.

14. Chopin N, Floccard B, Sobas F, Illinger J, Boselli E, Benatir F, Levrat A, Guillaume C, Crozon J, Négrier C, Allaouchiche B: Activated partial thromboplastin time waveform analysis: a new tool to detect infection? Crit Care Med 2006, 34:1654-1660

15. Zakariah AN, Cozzi SM, Van Nuffelen M, Clausi CM, Pradier O, Vincent JL: Combination of biphasic transmittance waveform with blood procalcitonin levels for diagnosis of sepsis in acutely ill patients. Crit Care Med 2008, 36:1507-1512.

16. Smith EY, Charles LA, Van Cott EM: Biphasic activated partial thromboplastin time waveform and adverse events in non-intensive care unit patients. Am J Clin Pathol 2004, 121:138-141.

doi:10.1186/cc8226

Cite this article as: Schneider $C P$, et al:: Activated partial thromboplastin time waveform analysis as specific sepsis marker in cardiopulmonary bypass surgery. Critical Care 2010, 14:104. 